\title{
Remdesivir in Pregnant Patients with Novel Coronavirus Disease 2019: Case Series
}

\author{
Renu Singh $^{1} \oplus \cdot$ Anjoo Agrawal $^{1} \cdot$ Mona Asnani $^{1}$ \\ Received: 6 August 2021 / Accepted: 26 October 2021 / Published online: 2 December 2021 \\ (c) Federation of Obstetric \& Gynecological Societies of India 2021
}

Keywords COVID-19 $\cdot$ SARS-CoV-2 $\cdot$ Pregnancy $\cdot$ Remdesivir

\section{Introduction}

The devastating coronavirus disease 2019 (COVID-19) caused by severe acute respiratory syndrome coronavirus 2 (SARS-CoV-2) was declared a pandemic by the World Health Organization (WHO) in March 2020. Since then, more than 194 million cases and more than 4 million deaths have been reported to WHO [1]. The disease may present as a spectrum from asymptomatic disease to hypoxemic respiratory failure and death. The current literature suggests that pregnancy does not increase the susceptibility to SARSCoV-2 infection but appears to worsen the clinical course of COVID-19, when compared to age-matched non-pregnant women. Further, pregnant women with symptomatic confirmed SARS-CoV-2 infection had a higher risk of ICU admission, receiving invasive mechanical ventilation and death [2].

Remdesivir (GS5734) is a nucleotide analogue. It reduces the replication of SARS-CoV-2 in vitro through selective inhibition of viral RNA-dependent RNA polymerase which SARS-CoV-2 utilizes for its host cell replication. Few trials have shown the efficacy of Remdesivir in shortening the duration and severity of moderate and severe COVID-19 disease in adults [2]. Based on these, an emergency use

Renu Singh, Professor, Department of Obstetrics and Gynecology, King George's Medical University, Lucknow, India, 226003. Anjoo Agarwal, Professor, Department of Obstetrics and Gynecology, King George's Medical University, Lucknow, India, 226003. Mona Asnani, Associate Professor, Department of Obstetrics and Gynecology, King George's Medical University, Lucknow, India, 226003.

Renu Singh

narayanrenu@yahoo.com; renusingh@kgmcindia.edu

1 Department of Obstetrics and Gynecology, King Georges Medical University, Uttar Pradesh, Lucknow 226003, India authorization of Remdesivir was granted by the United States Food and Drug Administration and Ministry of Health and Family Welfare, India [3]. Data regarding treatment of COVID-19 in pregnant women are limited. Here, we report four pregnant women hospitalized at our institution with confirmed SARS-CoV-2 infection, successfully treated with Remdesivir.

\section{Case 1}

A 29-year-old woman, day 1 post-cesarean, presented with fever, cough and difficulty in breathing. Her medical history was unremarkable except mild anemia. On admission, she had a temperature of $100.7^{\circ} \mathrm{F}$ and blood pressure of $140 / 90 \mathrm{mmHg}$ and was tachypneic to 26 breaths per minute with $88 \%$ oxygen saturation on room air. The hypoxia improved with supplemental oxygen $(10 \mathrm{~L})$ by nasal cannula and later by non-rebreather mask (NRM). Her chest $\mathrm{X}$-ray demonstrated bilateral hazy opacities suspicious for COVID-19 pneumonia. The laboratory results showed leukocytosis with neutrophilia, elevated CRP, LDH and d-dimer (Table 1). She tested positive for COVID-19. The patient met the criteria for severe COVID-19 and received five doses of Remdesivir, as per our institution protocol (Table 2), after consultation with the infectious disease specialist. An informed consent was obtained. She completed the course of Remdesivir successfully without any side effects. She was gradually weaned off from oxygen to room air on day 6 and was discharged on day 7 of hospital admission. 
Table 1 Laboratory values of patients with severe COVID-19

\begin{tabular}{|c|c|c|c|c|c|c|c|c|}
\hline \multirow[t]{2}{*}{ Laboratory parameters } & \multicolumn{2}{|l|}{ Case 1} & \multicolumn{2}{|l|}{ Case 2} & \multicolumn{2}{|l|}{ Case 3} & \multicolumn{2}{|l|}{ Case 4} \\
\hline & Admission & Discharge & Admission & Discharge & Admission & Discharge & Admission & Discharge \\
\hline WBC $\left(4.0-11.0 \times 10^{3} / \mathrm{ul}\right)$ & 21,700 & 15,300 & 16,300 & 13,500 & 15,600 & 10,800 & 9400 & 8300 \\
\hline Lymphocytes (\%) & 10 & 15 & 10 & 10 & 11 & 11 & 10 & 07 \\
\hline $\mathrm{Hb}(11.9-15.0 \mathrm{~g} / \mathrm{dl})$ & 8.3 & 8.3 & 11.8 & 10.9 & 10.5 & 11.6 & 9.0 & 8.9 \\
\hline AST (10-40 IU/L) & 74.6 & 47.8 & 44.0 & 20.3 & 73.2 & 83.7 & 98.7 & 42.9 \\
\hline ALT (10-40 IU/L) & 34.6 & 33.7 & 54.0 & 36.7 & 35.3 & 129.5 & 40.8 & 163.5 \\
\hline Alkaline phosphatase (50-240 IU/L) & 555.9 & 871.9 & 482.4 & 471.7 & 394.9 & 1112.3 & 813.9 & 502.4 \\
\hline \multicolumn{9}{|l|}{ Serum bilirubin } \\
\hline$(0.3-1.4 \mathrm{mg} / \mathrm{dl})$ & 1.05 & 0.75 & 0.55 & 0.32 & 0.87 & 0.49 & 1.04 & 0.53 \\
\hline $\mathrm{CRP}(<6 \mathrm{mg} / \mathrm{dl})$ & 61.6 & 19.1 & 3.6 & 149.9 & 66.7 & 1.7 & 78.1 & 20.6 \\
\hline D-Dimer $(<0.5 \mathrm{ug} / \mathrm{ml})$ & 2.3 & 0.9 & 0.8 & 0.6 & 0.62 & 1.05 & 0.5 & 1.01 \\
\hline Ferritin $(13.0-150.0 \mathrm{ng} / \mathrm{ml})$ & 96.9 & 72.2 & 234.9 & 57.9 & 74.7 & 13.3 & 188.7 & 88.9 \\
\hline LDH (240-480 IU/L) & 1321.9 & 1529.3 & 899.4 & 717.1 & 1189.9 & 647.5 & 1186.9 & 571.6 \\
\hline Creatinine $(0.5-1.4 \mathrm{mg} / \mathrm{dl})$ & 1.19 & 0.7 & 1.3 & 0.72 & 0.6 & 0.58 & 0.76 & 0.55 \\
\hline
\end{tabular}

WBC White blood cell count; $H b$ hemoglobin; $A S T$ aspartate aminotransferase; $A L T$ alanine aminotransferase; $C R P$ C-reactive protein; $L D H$ lactate dehydrogenase

Table 2 Remdesivir dosing protocol

\begin{tabular}{ll}
\hline Day & Dose of Remdesivir \\
\hline Day1 & Loading dose of $200 \mathrm{mg}$ \\
& intravenously(IV) as single \\
& dose \\
Days 2-5 & $100 \mathrm{mg}$ IV daily
\end{tabular}

\section{Case 2}

A 30-year-old primigravida at 40 weeks of pregnancy with preeclampsia and confirmed SARS-CoV-2 infection was referred. She was asymptomatic on admission. On admission, she was afebrile with BP of 150/98 $\mathrm{mmHg}$, with $98 \%$ oxygen saturation on room air. She underwent emergency cesarean delivery and delivered a baby boy weighing $3.2 \mathrm{~kg}$. The baby tested COVID negative and did not receive breast milk due to paucity of Remdesivir safety data.

On postoperative day 2 , she complained of breathlessness, requiring oxygen supplementation on a mask at $2 \mathrm{~L}$ to maintain Spo2 $>95 \%$. The chest X-ray showed bilateral hazy infiltrates, more on the left lower lobe. On day 3 post-cesarean, her breathlessness worsened requiring $5 \mathrm{~L}$ oxygen by mask. The laboratories were notable for mildly raised transaminases, raised serum ferritin, CRP and LDH levels. The patient received five doses of Remdesivir after informed consent. She completed the dosage of Remdesivir uneventfully. The inflammatory parameters showed a declining trend (Table 1). She was gradually weaned off from oxygen supplementation to room air on day 8 and was discharged on day 9 of admission.

\section{Case 3}

A 32-year-old, G2P1L1 at 25 weeks of gestation, presented with fever, cough and breathlessness. She was tested COVID positive and hence referred. On admission, her temperature was $101^{\circ} \mathrm{F}$, her $\mathrm{BP}$ was $122 / 76 \mathrm{mmHg}$, and she was tachypneic to 28 breaths per minute. Her medical history was unremarkable except hypothyroidism. Her pregnancy had been uncomplicated so far. The oxygen saturation on room air was $89 \%$, requiring $5 \mathrm{~L}$ oxygen supplementation by NRM to maintain Spo2 $>95 \%$. X-ray chest showed bilateral diffuse opacities suspect for COVID pneumonia. On day 2 of admission, her oxygen requirement increased to $15 \mathrm{~L}$ on NRM, and her inflammatory markers showed an upward trend. She was shifted to an intermediate care unit. She received five doses of Remdesivir after informed consent. During the therapy, her serum transaminases peaked on day 5 of starting Remdesivir but were never more than five times the upper limit of normal and the inflammatory markers declined (Table 1). During her ICU stay, fetal Doppler was used to monitor the fetal status.

On day 7 of admission, her oxygen requirement declined gradually from 15 to $3 \mathrm{~L} /$ minute by NRM. On day 10 , she was shifted to the ward with $2 \mathrm{~L}$ of oxygen supplementation to maintain Spo $2>95 \%$. During her stay in the ward, she remained afebrile, asymptomatic and perceiving fetal movements. An obstetric ultrasound showed a normal fetal scan. She was discharged on day 16 of admission with appropriate advice. 


\section{Case 4}

A 34-year-old, G3P1L1, admitted at 33 weeks of gestation and previous LSCS with fever and features of severe acute respiratory infection. On admission, she had a temperature of $101^{\circ} \mathrm{F}, \mathrm{BP}$ of $100 / 60$ and tachypneic to 28 breaths per minute, oxygen saturation of $86 \%$ on room air. Her oxygen saturation improved to $93 \%$ on NRM at 14 L. Her nasopharyngeal swab tested positive for COVID-19. Her chest $\mathrm{X}$-ray showed infiltrates, more on the right side. She was shifted to ICU on day 2 of admission following increased oxygen requirements of $20 \mathrm{~L}$ with failing oxygen saturation to $90 \%$. She underwent an emergency cesarean section in view of deteriorating maternal condition. She delivered a baby girl weighing $1.8 \mathrm{~kg}$ with Apgar of 7/9. She received five doses of Remdesivir, after informed consent uneventfully as per our institution protocol. The baby tested COVID negative and did not receive breast milk due to paucity of safety data.

In the immediate postoperative period, her $\mathrm{Spo} 2$ was $95 \%$ at $20 \mathrm{~L}$ oxygen. Her laboratory parameters showed an improving trend (Table 1). Gradually on postoperative day 5 , her oxygen requirement decreased to $15 \mathrm{~L}$. On postoperative day 9, she was shifted out from ICU to ward. Her requirement for oxygen decreased gradually, and finally she was off oxygen, maintaining Spo $2>95 \%$ on room air on postoperative day 13. She was discharged with her baby on day 19 of admission.

\section{Discussion}

The current COVID-19 pandemic has resulted in an unprecedented global healthcare crisis. The pregnant women remain at high risk of adverse outcomes, as the COVID-19 pandemic continues. Safe and effective therapy is crucial in these women. In all our cases who required supplemental oxygen, initiation of Remdesivir led to resolution of oxygen requirement and recovery from illness. The largest study on compassionate use of Remdesivir in pregnant hospitalized patients $(n=67)$ showed that $93 \%$ women recovered from their illness despite $67 \%$ requiring ICU admission. Remdesivir was well tolerated, and the incidence of serious adverse events was $18 \%$. However, the majority of the adverse events were related to pregnancy and/or the underlying disease. Other studies regarding outcomes following Remdesivir use in pregnancy have been limited to a few case series [2]. Of the 13 hospitalized patients, $61.5 \%$ were admitted in ICU; however, all recovered from their illness. Mild transaminitis was the only adverse effect noted [2].

Indian data in relation to treatment of severe COVID-19 infection in pregnancy with Remdesivir are sparse. However, the patients recovered well without any major side effects and had favorable outcome [4]. The available data till date support good tolerability and efficacy of Remdesivir in pregnant women with COVID-19.

Acknowledgements We thank all the pregnant women, doctors, nurses and other staff for the support and care of COVID-19-positive pregnant women.

Funding The authors received no financial support for the case series.

\section{Declarations}

Conflict of interest The authors declare that they have no conflict of interest.

Ethics approval This study involving human participants was performed in accordance with the ethical standards of the Institutional Ethics Committee of King George's Medical University, Lucknow.

Informed Consent Informed consent was obtained from individual participants included in the study.

\section{References}

1. World Health Organization. COVID-19 weekly epidemiological update. 2021 July 27 [cited 2021 July 29]; https://www.who.int/ covid-19

2. Lampejo T. Remdesivir for the treatment of COVID-19 in pregnancy. J Med Virol. 2021;93:4114-9. https://doi.org/10.1016/j. ajog.2020.08.001.

3. Advisory for Rational use of Remdesivir for COVID-19 Treatment. 2021 July 1 [cited 2021 July 1]. https://www.mohfw.gov.in

4. Singh V, Choudhary A. Treatment with Remdesivir in two pregnant patients with Covid-19 pneumonia. Cureus 13(5): e14986. Doi: https://doi.org/10.7759/cureus.14986.

Publisher's Note Springer Nature remains neutral with regard to jurisdictional claims in published maps and institutional affiliations.

\section{About the Author}

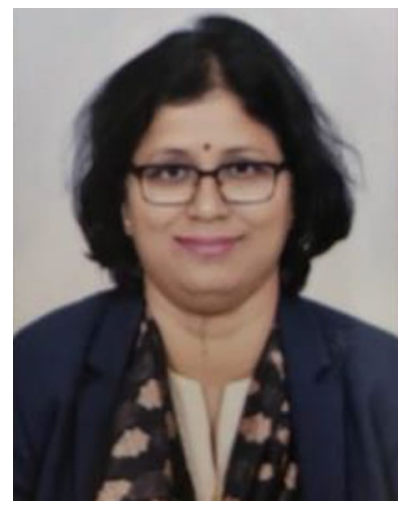

Dr. Renu Singh is working as Professor in Department of Obstetrics and Gynecology, King George's Medical University, Lucknow, a tertiary level referral hospital.Her area of interest is high risk pregnancy. 